\title{
MODELLING OF POWER CONVERTERS USING Z-TRANSFORM
}

The paper introduces a novel method of analysis and modelling of power electronic converters. Z-transform, numerical series (sequences) are used for both steady and transient states investigation of converters. The new impulse switching functions are created which are used as exciting functions of one- and multidimensional state-space models. Theoretical waveforms are compared with simulation results.

Keywords: Power converter, impulse switching function, Z-transform, inverse Z-transform, modelling and simulation, steady state operation.

\section{Introduction - Impulse switching functions}

There are many methods of analyses of power converters. Classical analytic methods, Laplace transform or/and Fourier analysis are suitable mainly for steady state operation [1], [2]. Transient analysis uses dynamic state-space modeling and/or Z-transform method. One of the fastest methods is that, which uses impulse switching functions (ISF). This method is used in signal theory, lesser in electrical engineering. Obviously, caused by power converters nature, those ISFs are strongly non-harmonic; sometime piece wise constant with zero spaces between pulses [3]. Then, it deals with power series of time pulses. From those series the impulse switching functions can be derived which are again orthogonal ones. Derived relations for voltages sequences can be used for current sequences calculations in electrical engineering system using impulse transfer function of the used plant and its time discretization. Sim- ilarly, one can derive relation for continuous time functions of voltages and currents. They are often grouped in two orthogonal $\alpha$ and $\beta$ axes [4], [5].

Examples of impulse switching functions belonging to output voltage of single- and three-phase inverters are shown in Fig. 1a and $1 \mathrm{~b}$, respectively.

\section{Mathematical description of ISFs using Z-transform}

Converter output phase voltages in Z-domain

Using basic definition of Z-transform (linearity-, shift right, index exchanging theorems) - and taking into account Z-images of constant- and alternating series [6] we can write

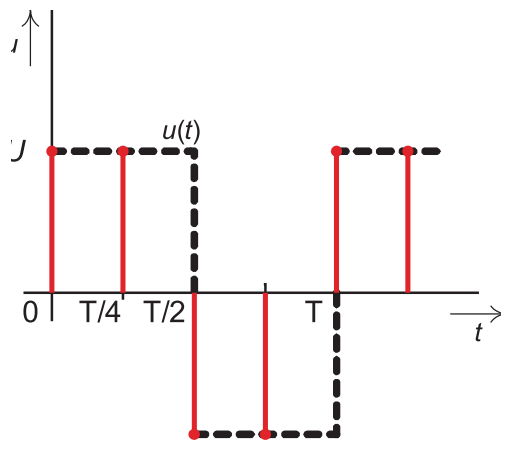

a)

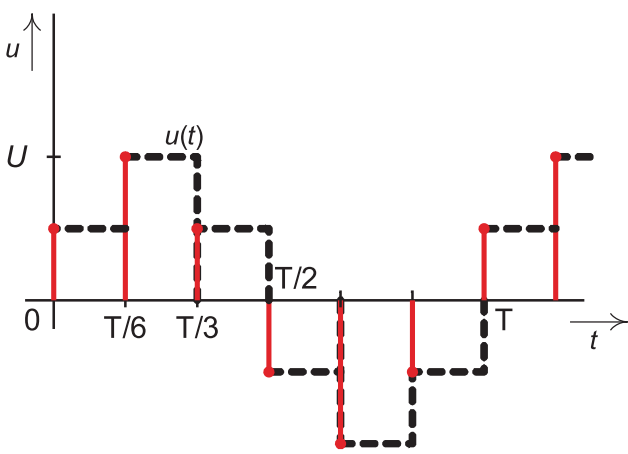

b)

Fig. 1 Impulse switching functions of single a)- and three phase b) voltage source inverters (VSI)

\footnotetext{
* Branislav Dobrucky ${ }^{1}$, Michal Pokorny ${ }^{1}$, Mariana Benova ${ }^{1}$, Mahmud Ali Rzig Abdalmula ${ }^{2}$

${ }^{1}$ Faculty of Electrical Engineering, University of Zilina, Slovakia, E-mail: branislav.dobrucky@fel.uniza.sk

${ }^{2}$ University of Zawia, Libya
} 
- for single/two-phase $\alpha, \beta$-system

$$
\begin{aligned}
& U_{\alpha}(z)=U \frac{z^{2}+z}{z^{2}+1} \text { and } \\
& U_{\beta}(z)=-U \frac{z^{2}+z}{z^{2}+1},
\end{aligned}
$$

where $U_{a}(z), U_{\beta}(z)$ are voltages in orthogonal axes and roots of polynomial of denominator are

$$
z_{1,2}= \pm j= \pm(-1)^{\frac{1}{2}}=e^{ \pm \frac{\pi}{2}}
$$

placed on boundary of stability in unit circle [6], [7] see Fig. 2,

- for three-phase $\alpha, \beta$-system (i.e. transformed, orthogonal system)

$$
\begin{aligned}
& U_{\alpha}(z)=\frac{z^{3}+2 z^{2}+z}{z^{3}+1} \text { and } \\
& U_{\beta}(z)=-U \frac{z^{3}+z^{2}}{z^{3}+1}
\end{aligned}
$$

where roots of polynomial of denominator are

$$
z_{1,2}=\frac{1}{2} \pm j \frac{\sqrt{3}}{2}=e^{ \pm j \frac{\pi}{3}} ; z_{3}=-1 ;
$$

placed again on boundary of stability in unit circle [6], [7], Fig. 2.
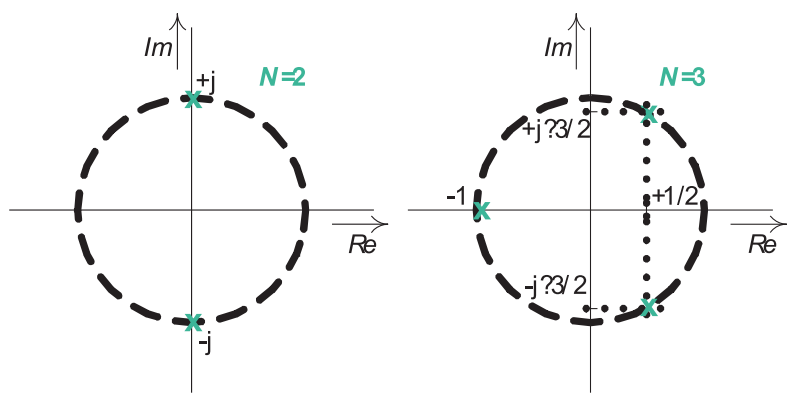

Fig. 2 Denominator poles placement of single a) - and three-phase z-form voltages in unit circle

Applying inverse Z-transform for converter output phase voltages in Z-domain we can create orthogonal impulse switching functions.

For inverse Z-transform $U_{(a, \beta)}(z) \leftrightarrow\left\{u_{n}\right\}$ one can use the residua theorem [7], [8]

$$
\sum_{i=1}^{N} \underset{z}{\operatorname{ess}} U(z) z^{n-1}=\sum_{i=1}^{N} \lim _{z \rightarrow z_{i}}\left(z-z_{i}\right) U(z) z^{n-1}
$$

where $n=0,1,2, \ldots ; N$ is number of poles.

or, if $U(z)$ can be expressed as ratio of polynomials of z-variable

$$
\sum_{i=1}^{N} \operatorname{res} U(z) z^{n-1}=\sum_{i=1}^{N} \frac{A\left(z_{i}\right)}{B^{\prime}\left(z_{i}\right)} z_{i}^{n-1},
$$

where $B^{\prime}(z)$ is the derivative of $B(z)$

$$
\frac{d B(z)}{d z}\left(\text { at } z=z_{i}\right) \text {. }
$$

Applying inverse Z-transform for single/two-phase $\alpha, \beta$-system

$$
\begin{aligned}
& U_{\alpha}(z)=U \frac{z^{2}+z}{z^{2}+1} \leftrightarrow\left\{u_{n}\right\}=\left\{u_{\alpha}\left(n \frac{T}{4}\right)\right\}= \\
& =\sum_{i=1}^{N} \lim _{1 \rightarrow z_{i}}\left(z-z_{i}\right) U \frac{z+1}{z^{2}+1} z^{n}
\end{aligned}
$$

after adapting

$$
\begin{aligned}
& u_{\alpha}\left(n \frac{T}{4}\right)=U \frac{1}{2}(-1)^{\frac{n}{2}}\left\{1+(-1)^{n}-\right. \\
& \left.-(-1)^{\frac{1}{2}}\left[1-(-1)^{n}\right]\right\}
\end{aligned}
$$

or

$$
u_{\alpha}\left(n \frac{T}{4}\right)=U \sqrt{2} \sin \left(n \frac{\pi}{2}+\frac{\pi}{4}\right) .
$$

By similar way for $\beta$-axis

$$
\begin{aligned}
& u_{\beta}\left(n \frac{T}{4}\right)=-U \frac{1}{2}(-1)^{\frac{n}{2}}\left\{1+(-1)^{n}-\right. \\
& \left.-(-1)^{\frac{1}{2}}\left[1-(-1)^{n}\right]\right\}=-U \sqrt{2} \cos \left(n \frac{\pi}{2}+\frac{\pi}{4}\right) .
\end{aligned}
$$

Applying inverse Z-transform for three-phase $\alpha, \beta$-system

$$
\begin{aligned}
& U_{\alpha}(z)=U \frac{z^{3}+2 z^{2}+z}{z^{3}+1} \leftrightarrow\left\{u_{n}\right\}=\left\{u_{\alpha}\left(n \frac{T}{6}\right)\right\}= \\
& =\sum_{i=1}^{N} \lim _{z \rightarrow z_{i}}\left(z-z_{i}\right) U \frac{z^{2}+2 z+z}{z^{3}+1} z^{n}
\end{aligned}
$$

after adapting

$$
\begin{aligned}
& u_{\alpha}\left(n \frac{T}{6}\right)=U \frac{1}{2}\left[(1-j \sqrt{3})(1+j \sqrt{3})^{n}+\right. \\
& \left.+(1+j \sqrt{3})(1-j \sqrt{3})^{n}\right]
\end{aligned}
$$

or

$$
u_{\alpha}\left(n \frac{T}{6}\right)=U_{D C} \frac{2}{3} \sin \left(n \frac{T}{3}+\frac{T}{6}\right) .
$$

By similar way for $\beta$-axis of three-phase converter

$$
u_{\beta}\left(n \frac{T}{6}\right)=-U_{D C} \frac{2}{3} \cos \left(n \frac{T}{3}+\frac{T}{6}\right) .
$$

Taking discrete state-space model for three-phase converter output current as state-variable considering the $1^{\text {st }}$ order load (resistive-inductive or resistive-capacitive)

$$
x_{n+1}=\boldsymbol{F}_{T / 6} x_{n}+\boldsymbol{G}_{T / 6}\left\{u\left(n \frac{T}{6}\right)\right\}
$$

where $\boldsymbol{F}_{T / 6}, \boldsymbol{G}_{T / 6}$ are fundamental and transition matrices (in general) of system parameters.

Applying Z-transform and considering the $1^{\text {st }}$ order load 


$$
\begin{aligned}
& z X(z)=F_{\tau / 6} X(z)+G_{\tau / 6} Y_{\infty}(z) \rightarrow X(z)= \\
& X_{\infty} G_{T / 6} \frac{z^{3}+2 z^{2}+z}{\left(z-F_{T / 6}\right)\left(z^{3}+1\right)},
\end{aligned}
$$

where $X(z)$ is image of founded state-variable (inductor current or capacitor voltage), and $Y_{\infty}(z)$ is maximum of steady state value ( $U / R$ or $U$, respectively).

$$
\begin{aligned}
& \text { And after adaption and simplification } \\
& X(z)=X_{\infty} G_{\tau / 6} \frac{z(z+1)}{\left(z-F_{T / 6}\right)\left(z^{2}-z+1\right)}= \\
& =X_{\infty} G_{\tau / 6} \frac{z(z+1)}{\left(z-z_{0}\right)\left(z-z_{1}\right)\left(z-z_{2}\right)},
\end{aligned}
$$

where

$$
z_{0}=F_{T / 6}, z_{1,2}=\frac{1}{2} \pm j \frac{\sqrt{3}}{2}=e^{ \pm j \frac{\pi}{3}}
$$

Applying inverse Z-transform

$$
\begin{aligned}
& x\left(n \frac{T}{6}\right) \equiv\left\{x_{n}\right\}=X_{\infty} G_{T / \%} \cdot \lim _{z \rightarrow z_{i}} . \\
& \left\{\sum_{i=0}^{2}\left(z-z_{i}\right) \frac{z_{i}\left(z_{i}+1\right)}{\left(z_{i}-z_{0}\right)\left(z_{i}-z_{1}\right)\left(z_{i}-z_{2}\right)} z_{i}^{n}\right\}
\end{aligned}
$$

Finally we get discrete form of state variable (converter output state variable - inductor currents or capacitor voltages)

$$
\begin{aligned}
& x\left(n \frac{T}{6}\right) \equiv\left\{x_{n}\right\}=X_{\infty} \frac{G_{T / 6}\left(1+F_{T / 6}\right)}{F_{T / 6}^{2}-F_{T / 6}+1} . \\
& {\left[F_{T / 6}^{n}+\sqrt{3} \frac{1-F_{T / 6}}{1+F_{T / 6}} \sin \left(n \frac{T}{3}\right)-\cos \left(n \frac{T}{3}\right)\right],}
\end{aligned}
$$

where $n=0,1,2, \ldots$

\section{Calculation of ISF function values using series and sequences}

The ISF functions presented above are numerical series (sequences) or trigonometric ones, respectively.

At first it is necessary to determine the $\boldsymbol{F}_{T / 6}, \boldsymbol{G}_{T / 6}$ functions values which are the state-variable values in 1/6-instant of time period (i.e. they are state- and transition responses). These can be obtained e.g. by using recursive relation for one-pulse solution:

$$
\frac{d x(t)}{d t}=A \cdot x(t)+B Y_{\infty}(t)
$$

thus recursive relation

$$
x(k+1)=\mathrm{F}_{\Delta} x_{k}+G_{\Delta} X_{\infty},(t)
$$

where $i_{k=0}=I_{0}=0$. Solution in z-domain yields

$$
X(z)=X_{\infty} \frac{G_{\Delta}}{\left(z-F_{\Delta}\right)} \frac{1-\left(r z^{-1}\right)^{60}}{\left(1-r z^{-1}\right)},
$$

where $F_{\Delta}$ a $G_{\Delta}$ are discrete impulse responses of state-variables gained by some of identification methods. The second fraction term is z-image of the partial sum of voltage impulses $(1 \div 60)$ [6], since $r^{a n} \cdot z^{-n} ; a<0$ is geometrical series, see Fig. 3 .
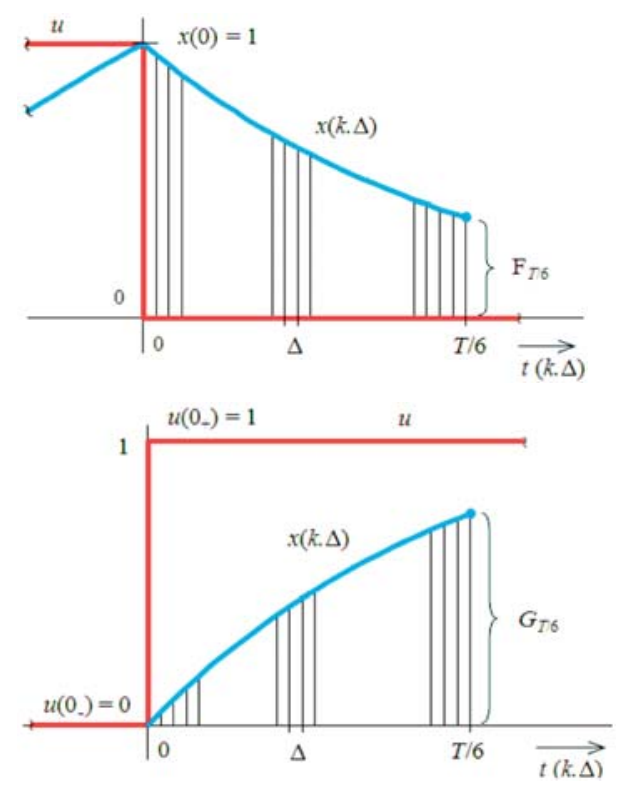

Fig. 3 To determination of $F_{T / 6}, G_{T / 6}$

After choosing $\Delta=T / 360$, $k$ will be the in the range of $0 \div 59$, thus $G_{T / 6}=y(60)$ and $F_{T / 6}=1-G_{T / 6}$. Supposing time constant of the load equal to $T / 2$ and

$$
F_{\Delta}=0.9944598 ; G_{\Delta}=0.0055401
$$

those values of $F_{T / 6}, G_{T / 6}$ will be

$$
F_{T / 6}=F_{\Delta} q^{N-1}=F_{\Delta}^{60}=0.71652923,
$$

because of $q=F_{\Delta}$.

$$
G_{T / 6}=G_{\Delta} \frac{1-F_{\Delta}^{60}}{1-F_{\Delta}}=1-F_{\Delta}^{60}=0.2834707
$$

Now, one can calculate state-variable $x\left(n \frac{T}{6}\right)$ for any $n$, Fig. 4 .

It is also possible to change the step of series (sequences) e.g. for step equal $T / 2$, by determining of $F_{T / 2}$ and $G_{T / 2}$ :

$$
F_{T / 2}=F_{T / 6}^{3}=0.3678764,
$$

and regarding to $G_{T / 6}$ :

$$
\begin{aligned}
& x(T / 6)=F_{T / 6} x(0)+G_{T / 6} X_{\infty} \\
& x(T / 3)=F_{T / 6} x(T / 6)+G_{T / 6} 2 X_{\infty} \\
& x(T / 2)=F_{T / 6} x(T / 3)+G_{T / 6} 2 X_{\infty} \rightarrow \rightarrow G_{T / 2}=0.8352386 .
\end{aligned}
$$

So, then one can calculate 


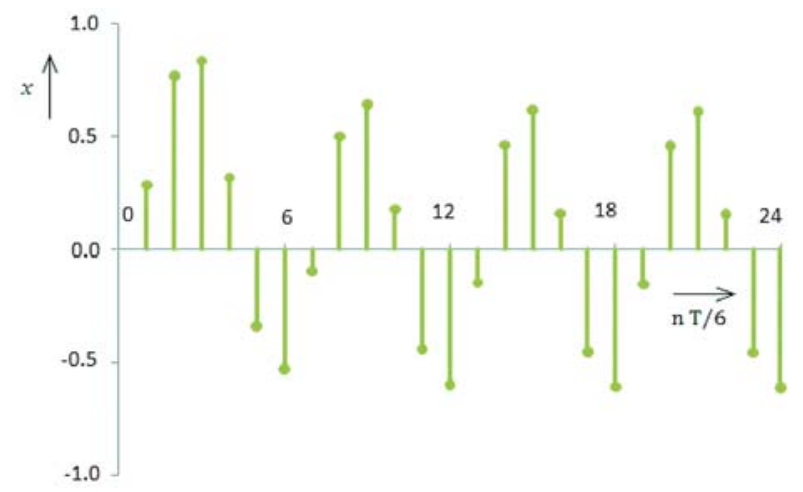
Fig. 4 Discrete response sequence of state-variable $\times\left(n \frac{T}{6}\right)$
of three-phase inverter

$$
x_{n+1}=F_{T / 2} x_{n}+G_{T / 2}\left\{u\left(n \frac{T}{2}\right)\right\}
$$

where $u\left(n \frac{T}{2}\right)=U(-1)^{n}$.

Steady state determination The steady state value of 'periodical' sequence $x\left(n \frac{T}{6}\right)$ can be
determined, based on condition

$$
x(0)=-x\left(\frac{T}{2}\right) \text { or vice versa,i.e. } x\left(\frac{T}{2}\right)=-x(0) .
$$

Then

$$
\begin{aligned}
& x\left(\frac{T}{2}\right)=F_{T / 2}\left[-x\left(\frac{T}{2}\right)\right]+G_{T / 2} X_{\infty} \rightarrow \\
& \rightarrow x\left(\frac{T}{2}\right)=\frac{G_{T / 2}}{1+F_{T / 2}} X_{\infty}=0.6106053 X_{\infty},
\end{aligned}
$$

so $x(0)=X_{0}=-0.6106053 X_{\infty}$.

Setting this value into $\left\{x_{n}\right\}$ or $x(n+1)$ we get:

$$
\begin{aligned}
& X_{0}=-0.6106053(n=0) ; \\
& X_{T / 6}=-0.1540492(n=1) ; \\
& X_{T / 3}=+0.4565561(n=2) ; \\
& X_{T / 2}=+0.6106053(n=3) .
\end{aligned}
$$

Based on zero order hold function [6] and total mathematical induction one finally yields:

For three-phase Clarke transformed system

$$
\begin{aligned}
& u_{\alpha}(k \Delta)=U_{D C} \frac{2}{3} \sin \left[\text { integer }\left(\frac{6}{T} k \Delta\right) \frac{\pi}{3}+\frac{\pi}{6}\right] \\
& u_{\beta}(k \Delta)=-U_{D C} \frac{2}{3} \sin \left[\text { integer }\left(\frac{6}{T} k \Delta\right) \frac{\pi}{3}+\frac{\pi}{6}\right] .
\end{aligned}
$$

where 'integer' means integer function, $T$ is time period and $k \Delta$ is discretizing time.

Derived relations for voltages can be used for state variable calculations in electrical engineering systems.

$$
x(k+1)=F_{\Delta} x_{k}+G_{\Delta} u_{\alpha, \beta}(k \Delta),
$$

where $F_{\Delta}$ a $G_{\Delta}$ are discrete impulse responses of state-variables (see above).

The steady state waveform of $\{x(k . \Delta)\}$ is shown in Fig. 5 .

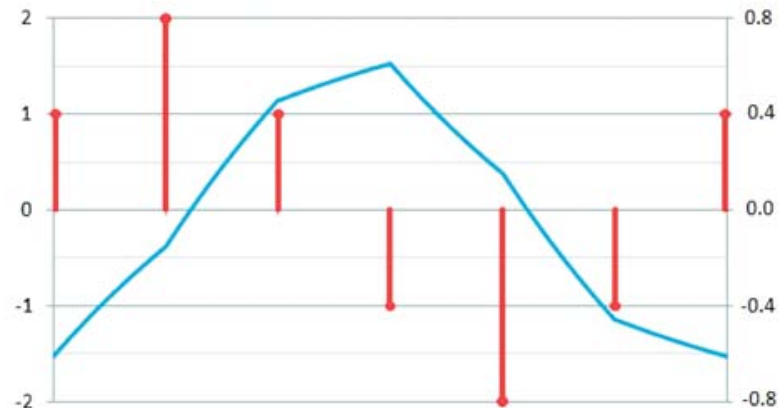

Fig. 5 Steady state waveform of state variable $x(k . \Delta)$

By application of above given approach it is possible to analyze model, and simulate any sample of output voltage of power voltage sourced inverter, like 12-pulse samples, Fig. 6.

As the last example, transient- and steady-state waveforms of state variable under sine PWM supply with minimum number of switching states (12-pulse switching voltage function) are shown in Fig. 7.

\section{Conclusions}

Using direct and inverse Z-transform and numerical series (sequences) it is possible to derive functions as discrete voltage sequences from output voltage of power converters. These impulse switching functions can then be used to express the state variables of electrical circuits which are connected to output of the converters. Presented techniques are suitable for both transient- and steady-state behavior investigations of power converters. Unlikely to pure numerical computing, ISFs make it possible to calculate variable values at any time instants.

\section{Acknowledgement}

The authors wish to thank for the financial support to Slovak Research and Development Agency project No. APVV-0138-10 and $R \& D$ operational program Centre of excellence of power electronics systems and materials for their components No. OPVaV-2008/2.1/01-SORO, ITMS 26220120046 funded by European regional development fund (ERDF). 


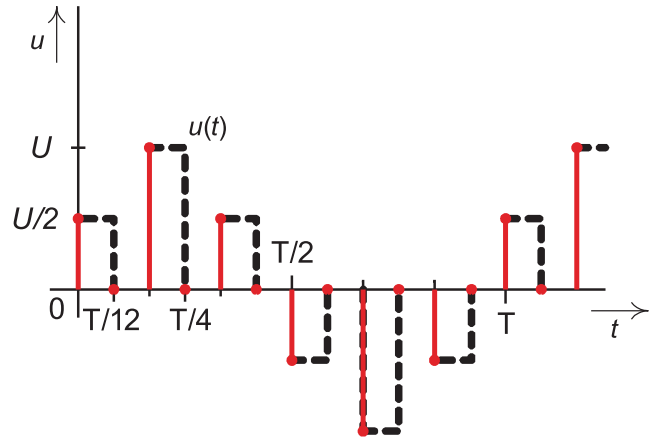

a)

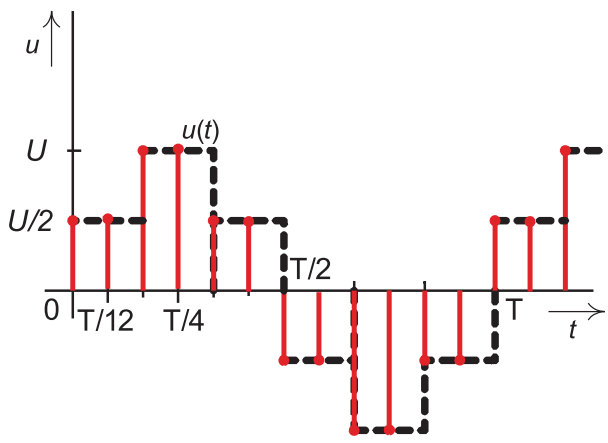

b)

Fig. 6 Impulse switching function of 12-pulse output voltage of 3-phase converter: a) - with half-width of impulses; b) - with half-step of the impulse sequence

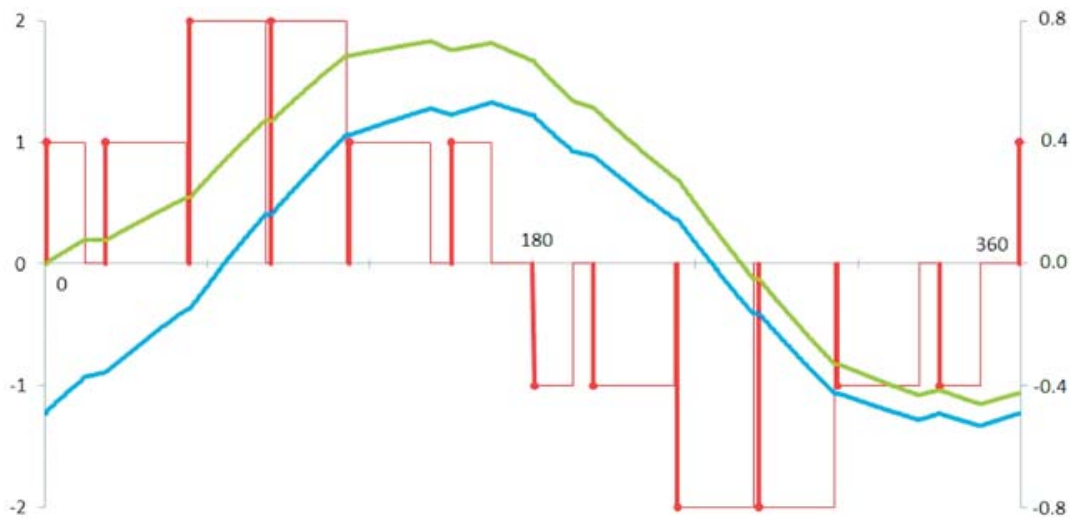

Fig. 7 State variable response under sine PWM supply

Legende: red - impulse switching function, blue - transient response, green - steady state waveform

\section{References}

[1] ZASKALICKA, M., ZASKALICKY, P., BENOVA, M., ABDALMULA, M. A. R., DOBRUCKY, B.: Analysis of Complex Time Function of Converter Output Quantities Using Complex Fourier Transform/Series. Communications - Scientific Letters of the University of Zilina, No. 1(12), pp. 23-30, 2010.

[2] DOBRUCKY, B., MARCOKOVA, M., POKORNY, M., SUL, R.: Using orthogonal and discrete transform for single-phase PES transients - a new approach. In: Modelling, identification, and control: Proceedings of the 27th IASTED Int'l Conference, Feb. 11-13, 2008, Innsbruck, Austria : Acta Press, 2008, ISBN 978-0-88986-633-1, pp. 60-65.

[3] JARDAN, R. K., DEWAN, B. S.: General Analysis of Three- Phase Inverters, IEEE Transactions on IGA, No. 6, pp. 672-679, 1969.

[4] DOBRUCKY, B., BENOVA, M., POKORNY, M: Using Theory of Series for Distortion Determination of Non-Harmonic Quantities of Two-Phase Systems. In: Int;l School on Non-Sinusoidal Currents and Compensation: Proceedings of the 10th conference-seminar, June 15-18, 2010, Łagów, Poland, IEEE, 2010, ISBN 978-1-4244-7894-1, pp. 37-42.

[5] GUldAN, V., MARCOKOVA, M.: Orthogonal Polynomials and Related Special Functions Applied in Geosciences and Engineering Computations. Communications - Scientific Letters, of the University of Zilina, vol. 12, No. 1, pp. 12-15, 2010.

[6] VICH, R.: Z-transformation and some of its Utilization (in Czech), SNTL Mathematical Seminar, Prague, 1983.

[7] ARAMOVIC, J. G., LUNC, G. L., ELSGOLC, L. C.: Functions of Complex Variable, Operator Calculus, Stability Theory (in Russian - translated into Slovak), Alfa, Bratislava, 1973.

[8] MORAVCIK, J.: Mathematical Analysis 3 (in Slovak), Textbook, Alfa Publishing House, Bratislava, 1992, ISBN 80-05-01044-3. 